\title{
Las complejidades del desarrollo socioeconómico en el caso de Andalucía
}

Pablo GALINDO CALVO

\section{RESUMEN}

A la hora de evaluar el desarrollo socioeconómico de una comarca, región, nación y/o sociedad, es necesario analizar y diagnosticar la indisociable relación que existe entre cultura -valores, creencias, normas, rituales, símbolos, normas y costumbres - y desarrollo socioeconómico. En el presente artículo abordamos los diferentes planteamientos teóricos que han analizado las diversas relaciones existentes entre factores culturales y el nivel de desarrollo socioeconómico de una sociedad con objeto de adoptar nuevas perspectivas de intervención y desarrollo futuros. Asimismo, presentamos los elementos claves que explican esta relación en el caso de Andalucía, en concreto, en lo referente a la cultura emprendedora imperante en su seno.

\section{PALABRAS CLAVE}

Valores, creencias, oportunidades vitales, móvil de logro, cultura productiva, desarrollo endógeno, bifurcación de valores, emprendedor, postmodernidad.

\footnotetext{
${ }_{1}^{1}$ Profesor de Sociología. Departamento de Sociología. Facultad de Ciencias Políticas y Sociología (Universidad de Granada). Profesor-Tutor Departamento de Sociología III (Tendencias Sociales). Universidad Nacional de Educación a Distancia (U.N.E.D.)
} 


\section{ABSTRACT}

At the time of evaluating the socio-economic development of a region, nation and/or society it is necessary to analyze and to diagnose indisociable relation that exists between culture -values, beliefs, norms, rituals, symbols and customs -and socio-economic development. In the present article we approach the different theoretical expositions that they have analyzed the diverse existing relations between cultural factors and the level of socio-economic development of a society with object to adopt new future perspective of intervention and development.

\section{EL IMPACTO DE LA CULTURA SOBRE EL DESARROLLO SOCIOECONÓMICO}

Hasta hace muy poco, cualquier realidad socioeconómica objeto de estudio se encontraba bajo el yugo de un análisis basado en los modelos de acción racional en los que la variable económica era la protagonista. El determinismo económico al que hemos estado abocados hasta hace muy poco ha ido desapareciendo de los diagnósticos y propuestas de intervención en las diferentes áreas de la vida social, económica y política. Por suerte, resulta cada vez más evidente que aquellos modelos que no tienen en cuenta los factores de índole cultural resultan a todas luces parciales e incompletos. Así, Guzmán Cuevas defiende la necesidad de que las políticas de desarrollo regional superen la visión eminentemente económica y financiera, ya que, por ejemplo, en el caso de Andalucía, es fundamental estudiar en profundidad los rasgos culturales y sociales de su población (1987). En otras palabras, todas aquellas interpretaciones de la realidad social que están basadas en el determinismo económico, cultural o político son demasiados simples ya que las conexiones casuales entre los diferentes factores son bidireccionales (Inglehart, en Díez Nicolás, 1994). Es más, autores como Inglehart, se muestran contrarios al mantenimiento de que la construcción cultural es el único factor que interviene en la formación de la experiencia humana, y por ello es tan importante mantener la interdisciplinariedad y la complementación factorial a la hora de diagnosticar la realidad social.

Si hacemos un breve repaso por los clásicos de la Sociología, encontramos que, para Weber, la relación existente entre cultura, economía y sociedad es la clave de la sociedad moderna. A juicio de este autor, son las ideas de la vida social 
y su significación cultural los elementos clave del complejo entramado humano y social (en Picó, 1999). Weber opinaba que eran los intereses materiales y no las ideas los que determinaban los comportamientos sociales, pero al mismo tiempo, también presentaba ejemplos de la influencia que las diferentes visiones del mundo -creencias- habían tenido sobre la acción humana y sus intereses² (Picó, 1999).

En la sociedad industrial avanzada, el determinismo económico es cada vez menos creíble. Cuando comenzó el proceso de industrialización en Occidente, el determinismo económico de Marx tuvo una buena acogida, la situación cultural de aquel entonces lo hacía, si cabe, más real, y el carácter univariante del mismo raspaba las posibles ambigüedades que surgieran en la explicación de la realidad (Inglehart, 1991). En la actualidad, el factor económico es importante pero ya no es crítico, son los factores culturales los que vienen a definir la actual realidad social. Para Marx, la cultura a través de su manifestación ideológica es la consecuencia de las relaciones sociales entre personas y grupos humanos cuyo resultado más representativo lo encontramos en la estructura social que de ella se deriva (Picó, 1999). Es más, hasta los conocidos trabajos de McClelland sobre la motivación por el logro, apenas si se daba importancia a otros factores que no fueran los puramente económicos, dejando de lado la enorme importancia que los factores políticos y, sobre todo, psicosociológicos juegan en la aparición y consolidación del desarrollo socioeconómico.

\footnotetext{
2 En este artículo no trataremos la obra de Weber "La Ética Protestante y el espíritu del Capitalismo". No obstante, hacemos alusión a sus aspectos fundamentales, ya que constituyen un hito fundamental a la hora de hablar la interacción entre cultura y desarrollo socioeconómico. Según Weber, el espíritu capitalista, el espíritu de empresa, mantenía una estrecha relación con una corriente específica de la teología protestante -el calvinismo- que eliminó la magia de la religión, enfatizó la vocación profesional e hizo del trabajo y del ahorro una señal de predestinación como parte de un intercambio con la divinidad o sus agentes sobre la tierra. Todo ello se tradujo en una acumulación y transmisión intergeneracional de la riqueza como algo digno de encomio y no de culpa (Buchanan, 1995). A juicio de Weber, son las ideas de la vida social y su significación cultural los elementos clave del complejo entramado humano y social (Picó, 1999). Weber opinaba que eran los intereses materiales y no las ideas los que determinaban los comportamientos sociales, pero al mismo tiempo, también presentaba ejemplos de la influencia que las diferentes visiones del mundo habían tenido sobre la acción humana y sus intereses. La idea fundamental de la tesis de Weber se refiere a que la cultura no es un fenómeno determinado por la economía, sino que entre ambos factores existe una interacción, una veces son los factores económicos los que marcan el curso de los factores culturales y viceversa. Centrándonos en la aportación específica con relación a nuestro tema, Weber afirmó que el protestantismo calvinista había evolucionado progresivamente hacia un sistema de valores que consideraba la acumulación de la riqueza por ella misma como un signo de gracia divina, y esto animó a un control ascético que llevó a la acumulación de riqueza, lo que a su vez dio lugar a un espíritu empresarial y una acumulación de capital (Inglehart, 1991). Todo ello se tradujo en un proceso de cambio que facilitó la Revolución Industrial y, en consecuencia, un mayor desarrollo socioeconómico en su globalidad genera mayor progreso económico (Buchanan, 1995).
} 
La gran diferencia entre Marx y Weber consiste en que para el segundo la influencia de las ideas y las creencias sobre las personas es muy fuerte, se presenta independiente de otras fuerzas sociales y su poder es, al menos, similar al de las fuerzas materiales (Picó, 1999). Para Marx, en cambio, la cultura existente se deriva de los procesos productivos imperantes en la sociedad y, por tanto, varía en función del momento histórico en que nos encontremos. En cambio, para Weber, la cultura no es un subproducto del capitalismo, sino todo lo contrario, determinadas ideas y creencias han sido las precondiciones culturales para el nacimiento del capitalismo.

A nuestro juicio, las claves económicas o culturales determinan de manera desigual la resolución de las diversas situaciones, siendo, en muchas ocasiones, las culturales las que mayor influencia ejercen de cara al mantenimiento 0 desenlace de las circunstancias que caracterizan cada situación. No se puede obviar que los factores económicos, así como los de carácter normativo y legislativo, marcan las relaciones empresariales, financieras, bursátiles y laborales con un alto grado de influencia. No obstante, existen otros planteamientos de influencia y determinación que deben ser tenidos en cuenta. No hay que discutir, ni mucho menos, la importancia y la influencia de la estructura y coyuntura económica sobre el desarrollo en general, pero no todo el esfuerzo debe ir encaminado a su análisis y la aplicación de su recetario (Cañadilla, 2000). Multitud de actuaciones en el ámbito del empleo y el desarrollo de los recursos humanos, de índole financiera, técnica, de gestión, de información, etc., sucumben 0 ven seriamente mermada su eficacia por causa del entorno sociocultural en el que se desarrollan: cultura o subculturas en las que muestran especial relevancia determinadas ideas y comportamientos de carácter pasivo, irresponsable, irrespetuoso, inmovilista, deshonesto. Estos factores culturales son un auténtico handicap para la evolución de la economía, el empleo, las empresas, la innovación, el desarrollo de los recursos humanos, el respeto al medio ambiente, la calidad, etc., (lbídem). Por suerte, los planteamientos eminentemente racionalistas y economicistas ya van reconociendo poco a poco la importancia de los factores sociales, culturales y psicológicos en el desarrollo, aunque le otorgan al factor económico el papel protagonista. En cualquier caso, son cada vez más los que afirman que, con relación a la situación de los países subdesarrollados, los factores irracionales sociales y psicológicos tienen tanta importancia que los programas de ayuda al desarrollo no deben diseñarse teniendo únicamente en cuenta el planteamiento puramente económico (McClelland, 1973). 
La propia cultura de la comarca, región, provincia o nación objeto de diagnóstico e intervención determina también la naturaleza de las relaciones sociolaborales y económicas. Cuando se habla de cultura, no se alude al pasado histórico o patrimonial de la zona, sino más bien a las costumbres, rituales, valores, creencias y actitudes que priman y que rigen el comportamiento de los individuos, grupos y organizaciones de esa comunidad o región. Es más, son numerosos los factores psicosociales y culturales que determinan el comportamiento de la población activa y dichos factores interactúan conjuntamente con la estructura y coyuntura económica ejerciendo una influencia mutua entre ambos.

A este respecto, nos vale como apunte introductorio el repaso que Irma Adelman hace de las grandes teorías -Adam Smiht, David Ricardo, Karl Marx, Schumpeter y Keynes- que explican el desarrollo económico de una sociedad. Así, Schumpeter afirma que la tendencia a la innovación por parte de las empresas no se debe únicamente a la capacidad de acumulación de la empresa, sino que depende del carácter emprendedor de los empresarios, del ambiente socio-cultural que rodea los esfuerzos empresariales y del espíritu de reconocimiento social del talento emprendedor (en Adelman, 1964). En otras palabras, un empresario puede tener una gran capacidad para introducir nuevas tecnologías y nuevos elementos técnicos en el proceso productivo, pero su mentalidad y los valores que rigen la cultura de su empresa pueden ser contrarios al comportamiento innovador. De igual manera, Adelman concluye en su libro que "el subdesarrollo no puede achacarse únicamente a la deficiencia de capital o a la falta de talento de empresa, o a una proporción población-recursos adversa, o a un ambiente institucional poco apropiado(...) En lugar de eso, el fenómeno del subdesarrollo debe entenderse en el contexto del total complejo de interrelaciones que caracterizan la vida económica y social de la comunidad" (Adelman, 1964:166).

Asimismo, no debemos de olvidar que igualmente también ha sido objeto de un largo proceso de estudio la relación existente entre condiciones culturales y psicológicas y la cultura democrática de una nación (Almond y Verba, 1970: 299). Desde Aristóteles hasta Marx, pasando por Weber, o autores como Lipset, Laswell 0 Rosenberg, se ha estudiado la relación entre las formas de la sociedad y el sistema político existente. Es decir, bajo el juicio de estos autores, las actitudes políticas de los ciudadanos están condicionadas por el ambiente social e interpersonal que les 
rodea. Así por ejemplo, en el estudio presentado por Lasswell, Rosenberg y Lane ${ }^{3}$ (en Almond y Verba, 1970) concluyen que en las democracias estables existe una mayor interacción social, se concede una mayor importancia a la colaboración y cooperación entre individuos, y se valoran más las cualidades de carácter exterior. Asimismo, a mayor confianza y seguridad, mayor es la estabilidad e intensidad democráticas, la confianza social es mayor en la sociedades con buena salud democrática, la cooperación cívica es mayor en las sociedades democráticas y la comunicación es más abierta y libre. De esta manera queda patente la necesidad de reforzar determinados valores y actitudes sociales con vistas a consolidar la salud democrática de una nación o incluso crear nuevos valores sociales con objeto de implantar un sistema democrático en un entorno, a priori, hostil al mismo. Pero es que, igualmente, nos encontramos con la otra cara de la moneda -realidad que garantiza a todas luces la naturaleza bidireccional de las relaciones aquí expuestas-, que no es otra que la estrecha relación existente entre desarrollo económico, niveles de educación y democracia. Dicho con otras palabras, existe una importante correlación entre modernización socioeconómica y el surgimiento de nuevas democracias (Fukuyama, 1992).

Con lo dicho anteriormente se desprende la idea de que las estrategias dirigidas a la mejora y reajuste de la vida socioeconómica y laboral -y política, si fuera el casode la población activa en particular y de la sociedad en general, deben tener en cuenta la doble realidad, objetiva -coyuntura económica- y subjetiva -cultura-, que determina su nivel de desarrollo socioeconómico, tanto para el diagnóstico de las causas y problemas como para la definición de las estrategias de intervención encaminadas a afrontar la evolución y necesaria readaptación del sistema (Cañadilla, 2000). En definitiva, el desarrollo económico no puede entenderse como un simple incremento del producto nacional -aumento de la eficacia-, sino que debe centrarse también en el desarrollo de la dimensión cultural -eficiencia y consistencia- (Argandoña, 1994).

Cañadilla, en el "Manual-Guía para el fomento de la Cultura E2", nos presenta la relación causal existente entre Cultura, en este caso Cultura E2 -EticoEmprendedora-, la confianza o la credibilidad y el desarrollo de las personas, grupos y organizaciones. Para este autor, en muchos casos, el éxito o fracaso

\footnotetext{
${ }^{3}$ En este estudio se analizaron los requisitos socio-psicológicos de la democracia en cinco naciones: Estados Unidos, Gran Bretaña, Alemania, Italia y México. Para más información consultar Lasswell, H. Power and Personality; Lane, E. Political Life, y Rosenberg, M. Misantrophy and Political Ideology.
} 
de un individuo en la búsqueda de su inserción laboral o la buena marcha de una empresa depende de la capacidad de estos de generar confianza y credibilidad en su entorno inmediato. Cuando un individuo, grupo u organización muestran respeto, responsabilidad, honestidad y constancia, están siendo depositarios de un alto nivel de confianza y credibilidad. Es en este momento cuando tienen ya en su poder una de las condiciones fundamentales para el desarrollo exitoso de su carrera profesional o empresarial (2000).

\section{EL CONCEPTO DE DESARROLLO}

El término Desarrollo, junto con el de Cultura, es otro de los conceptos que, gracias a su subjetividad, relatividad, complejidad y naturaleza comparativa, se convierte en otro de los grandes temas recurrentes a lo largo de la historia de las ciencias sociales.

Como nos recuerda Requena, el término Desarrollo es un concepto relativo (1999). Este carácter relativo proviene de comparar mediante un punto la posición más o menos desarrollada de una región, grupo, organización, comarca y/o sociedad. Para este autor, la medición del desarrollo de una organización, región o sociedad se basa en el grado de evolución de las estructuras sociales y culturales en las que éste tiene lugar.

Es complicado presentar una definición adecuada de lo que debemos entender por desarrollo socioeconómico. A juicio de Adelman, la enorme heterogeneidad, en cuanto a recursos, estructuras e instituciones que presentan las diferentes regiones y sociedades dificulta enormemente la tarea de definir los referentes que determinan cuándo se está en situación de subdesarrollo o desarrollo ${ }^{4}$ (1964).

\footnotetext{
${ }^{4}$ En opinión de Parsons los países desarrollados presentan, entre otras, las siguientes características: normas de logro, universalismo y especificidad. Por su parte, los caracterizados como subdesarrollados puntúan alto en normas adscriptivas, particularismo y difusividad (1982). El planteamiento de Parsons parte de las características que presenta la estructura social de la sociedad en cuestión con objeto de establecer diferencias entre las estructuras sociales de las sociedades modernas y tradicionales.
} 
En el presente trabajo, cuando hablamos de desarrollo nos estamos refiriendo, en un primer momento, a desarrollo personal, grupal, organizacional, empresarial, etc., pero en este caso, enfocado y dirigido hacia la consecución de otra de sus combinaciones, no menos interesante y de complejo entendimiento, el desarrollo socioeconómico.

Por otro lado, para definir el concepto desarrollo endógeno, nos remitiremos a la definición de Bericat Alastuey, que lo define como la "búsqueda racional de oportunidades vitales con sentido, susceptibles de ser alcanzadas mediante incrementos de riqueza" (1989:16). Como nos recuerda este autor, el desarrollo es un proceso de acción y por consiguiente se refiere a individuos, grupos, organizaciones y/o comunidades que tienen como objetivo común el aumento de sus oportunidades vitales. Pero para que haya desarrollo no basta con que se presenten estas oportunidades, sino que tienen que tener sentido y al mismo tiempo deben suponer un esfuerzo y estar socialmente legitimadas ${ }^{5}$ (Dahrendorf, 1983). En otras palabras, tiene que haber motivación y voluntad ${ }^{6}$. La otra parte de la definición hacía referencia a la generación de riqueza y ello supone el conocimiento por parte del sujeto de conocimientos racionales sobre el beneficio económico. Al mismo tiempo, es necesaria la confluencia de una estructura de recursos y una estructura de oportunidades en un contexto en el que las relaciones de influencia y poder entre los sujetos se tornan estratégicas (Bericat Alastuey, 1989). En resumen, voluntad, saber y poder son los tres ejes en torno a los que gira el desarrollo de los individuos, grupos y organizaciones.

Nuestro planteamiento en este trabajo viene a manifestar que existe una importante relación entre cultura y desarrollo socioeconómico ${ }^{7}$. Es decir, las diferentes formas y componentes culturales que conforman a una sociedad ejercen una notable influencia en el desarrollo y evolución de sus estructuras socioeconómicas. De esta manera, el desarrollo de las empresas, formadas por individuos y grupos, también está muy influenciado por los valores, creencias, normas y símbolos imperantes en

\footnotetext{
${ }^{5}$ El concepto ligaduras es el concepto utilizado por Dahrendorf para referirse a la conexión de sentido y finalidad que debe tener toda acción de desarrollo.

${ }^{6}$ Más adelante se verán los planteamientos de la Teoría Voluntarista del desarrollo.

${ }^{7}$ Los trabajos de Weber, McClelland, Inglehart, Adelman, Vernon o Wegnez nos dan muestra de ello.
} 
su entorno. Tal y como veíamos con anterioridad, volvemos a remitirnos a uno de los fragmentos clave de la obra de Cañadilla, que no viene sino a consolidar la enorme importancia que la cultura juega en los procesos de desarrollo, "postulamos que la conjunción de determinados valores de carácter ético-emprendedor, de las creencias que los sostienen y de las actitudes que generan -incorporados a la cultura de las personas y grupos que constituyen la población activa- propiciará comportamientos beneficiosos para el desarrollo personal, profesional y organizacional, así como para la mejora de las condiciones de empleo y del desarrollo socioeconómico en general "(2000:18).

Partiendo de la responsabilidad o no de la región en la búsqueda de su desarrollo, nos resulta fácil diferenciar dos grandes teorías del desarrollo, conforme a las cuales se pueden plantear adjetivaciones que no dejan lugar a dudas sobre la visión del desarrollo. En el primero de los casos, características como la pasividad, la desresponsabilización o el inmovilismo, nos describen el modelo de la teoría de la dependencia. Esta teoría viene a plantear que el subdesarrollo se debe a causas externas a la región y/o comunidad, individuo, grupo u organización. No se responsabiliza al sujeto y por tanto es fácil que el sujeto -o el grupo en cuestión- adopte una actitud victimista ante su situación de subdesarrollo. El aspecto más negativo de este planteamiento, a todas luces erróneo, es que se legitima la actitud resignada y dependiente del sujeto y por tanto los valores de la pasividad y la dependencia se tornan como grandes obstaculizadores de la iniciativa, la innovación, la creatividad, la cooperación, la proactividad o el dinamismo.

En contraposición con la teoría de la dependencia nos encontramos ante la teoría del desarrollo endógeno, planteamiento en el que el papel activo, responsable y cooperador del individuo, grupo, organización, región y/o comunidad caracterizan las actuaciones en pro de una situación de mayor desarrollo y por consiguiente de mayor riqueza. Bajo este planteamiento florecen valores como la participación, la innovación, la proactividad o la creatividad, valores que sin duda refuerzan el carácter emprendedor del sujeto.

Desde una perspectiva eminentemente economicista, nos apoyamos en los trabajos de Adelman para profundizar en la definición más económica, valga la redundancia, del término "desarrollo económico" (1964). Según esta autora, cuando hablamos del desarrollo económico de una comarca, región o sociedad, nos estamos 
refiriendo al proceso mediante el cual se transforma una economía cuyo ingreso por habitante presenta una tasa de crecimiento pequeña o negativa en una economía en la que el ingreso por persona tiene una tasa significativa de incremento autosostenido como una característica permanente a largo plazo. Por tanto, y desde este punto de vista, una región o sociedad es considerada como subdesarrollada cuando su desarrollo económico es posible pero incompleto. De esta manera, tal y como veíamos con anterioridad en la teoría de la dependencia, "la definición presentada sirve para separar el desarrollo económico de procesos tales como el crecimiento esporádico y el crecimiento sostenido principalmente por fuerzas exógenas" (Adelman, 1964:67).

Por último, y antes de adentrarnos en algunos de los planteamientos sociológicos que explican el desarrollo, debemos referirnos a las consecuencias negativas u obstáculos que se derivan del inicio y ejecución de un proceso de desarrollo socioeconómico. El desarrollo no supone solamente un cambio económico, sino que supone un cambio en las estructuras, en la comunidad y en los individuos. Ello se traduce en un cambio ocupacional para muchas personas y ello, como es sabido, genera un cambio de rol y, por tanto, de identidad social. Por ello nos encontramos ante un coste no solo económico, sino también social, material, simbólico y expresivo por parte de los sujetos que se implican en un proceso de desarrollo.

Desde un punto de vista más teórico y al mismo tiempo más crítico, haremos mención a dos grandes teorías del desarrollo, la Teoría Voluntarista del Desarrollo y la Teoría de la Bifurcación Posmoderna (Requena 1999). La primera de ellas hace hincapié en la importancia de la voluntad en todo proceso de desarrollo: si no hay voluntad no hay desarrollo. La intensidad y orientación de esta voluntad a la hora de acometer un proceso de desarrollo están determinadas culturalmente. En cambio, la segunda gran teoría nos señala que las sociedades contemporáneas desarrolladas están asistiendo a importantes cambios culturales que afectan a la intensidad y a la naturaleza de su voluntad de logro. En otras palabras, los ya tradicionales valores objetivos, materialistas y economicistas están siendo complementados con una serie de valores dirigidos al desarrollo personal y social y al enriquecimiento interior de la sociedad. Es decir, los valores típicos de la modernidad no han desaparecido, sino que están conviviendo con nuevos valores de naturaleza más social o postmaterial. 


\section{LA RELACIÓN EXISTENTE ENTRE CULTURA Y DESARROLLO SOCIOECONÓMICO}

Con todo lo dicho hasta ahora, ante preguntas tales como ¿qué relación existe entre cultura y desarrollo? o ¿qué importancia presentan los factores culturales en los modelos de desarrollo?, debemos contestar con absoluta rotundidad que la relación es alta y la importancia muy grande. Ello es debido a una justificación muy simple: si el desarrollo endógeno huye de las justificaciones externas a la hora de explicar su nivel de desarrollo, son los factores internos los que explican dicha situación, entre ellos, por supuesto, la cultura de los individuos, grupos y organizaciones que anidan en la región y/o comunidad objeto de desarrollo. En palabras de Bericat Alastuey, "la cultura constituye la mediación consciente e inconsciente de la conducta, contiene el instrumental y la capacidad cognitiva, y define los horizontes comunitarios" (1984: 19). En este orden de cosas, la cultura, en muchos de los casos, es el factor que más influye en el desarrollo de los países y explica por qué unos se desarrollan más rápidamente y equitativamente que otros (Harrison, 1987). Queda aún mucho por hacer a la hora de diseñar programas de intervención y desarrollo en el seno de una región y/o comunidad ya que, desde nuestro punto de vista, se sigue invirtiendo poco en "cultura" . Con ello no nos referimos a espectáculos, arte y folklore, sino que nos referimos a que sigue existiendo una concepción de coste y no de inversión ante los factores culturales, ya que, es por todos sabido, que los cambios culturales son a largo plazo y sus transformaciones se dejan entrever poco a poco. En otras palabras, la rentabilidad en las inversiones soft -elementos no materiales- está cada vez más clara, pero la realidad demuestra que la tendencia se inclina por una inversión hard -elementos materiales-. (Bericast Alastuey, 1984).

Por todo ello, podemos decir que una región puntuará alto en desarrollo, siempre y cuando destaque en tres de las áreas culturales más importantes de cara a la consecución de un incremento de la riqueza. La primera de ellas es la voluntad social de desarrollo, es decir, que existan valores favorables al desarrollo. La segunda es el conocimiento orientador para la creación de riqueza, y en tercer

\footnotetext{
${ }^{8}$ En el mundo de la empresa ocurre algo bastante parecido. La mentalidad de muchos de los empresarios considera que el gasto en la gestión social y cultural de las personas es una pérdida de tiempo y dinero y que para nada genera rentabilidad o beneficio a la empresa. La inversión en cultura se considera como un coste y no como una inversión. Ello se debe, entre otros factores, a la mentalidad a corto palzo imperante en muchos de ellos.
} 
lugar, la percepción de los recursos potenciales, valores emprendedores tales como la creatividad, la innovación, la imaginación, la iniciativa o el logro?.

\subsection{La interpretación psicológica del desarrollo económico}

Ya hemos visto con anterioridad la destacada presencia que los factores sociales, culturales y psicológicos tienen en la explicación, análisis y reflexión del nivel de desarrollo socioeconómico de un grupo, organización, región y/o comunidad. Las interpretaciones económicas, biológicas y ambientales han soportado casi la totalidad de los análisis que sobre el desarrollo económico se han realizado. Asimismo, parecen haber quedado claras las numerosas e importantes razones que justifican la indiscutible presencia de los factores sociales, culturales y psicológicos en el desarrollo económico, y por tanto es de justicia ampliar la interdisciplinariedad con la que se trata y estudia la realidad económica, máxime aun cuando gran parte de nuestra historia ha estado sumida en el dominio de las interpretaciones racionales y materiales de la realidad humana.

La toma de decisiones en materia económica, al igual que en otras áreas de la vida del hombre, está precedida por el complejo y apasionante mundo de los valore ${ }^{10}$, las creencias y las actitudes ${ }^{11}$. Es más, tal y como nos recuerda McClelland, han sido precisamente los pensamientos irracionales los que a lo largo de la historia han justificado numerosos logros y aventuras empresariales (1968)

\footnotetext{
${ }^{9}$ Así, por ejemplo, en el caso de la región andaluza -objeto de múltiples investigaciones que estudian su grado de desarrollo socioeconómico con relación a la cultura de la población y su entorno-, se reconoce abiertamente que la capacidad de sacrificio, la actitud ante el trabajo, el esfuerzo personal, la frugalidad, el hábito de ahorro, la iniciativa, la aceptación de riesgos, la planificación y otras actitudes, han sido elementos deficitarios en su historia -elementos de naturaleza cultural, no material- y que al mismo tiempo son claves a la hora de garantizar las condiciones necesarias para el progreso de una región.

${ }^{10}$ La importancia que los valores juegan en el comportamiento social es tan grande que su tratamiento y análisis no debe ser obviado en ningún momento, ya que no tiene discusión que el ser humano no siempre se comporta de manera racional

${ }^{11}$ Parece estar claro que, en el fondo, la propensión a ahorrar o invertir no es, ni más ni menos, que una actitud. Esta actitud, a su vez, está precedida por una serie de creencias y valores.
} 
Una vez clara la importancia de los factores "blandos"12 en el desarrollo, debemos dedicar nuestro esfuerzo a identificar cuáles son estos factores. Algunos economistas, como Rostow, en los años cincuenta abogaban por una serie de elementos humanos o predisposiciones importantes para el desarrollo desde un punto de vista económico: desarrollo de las ciencias fundamentales, aplicación de la ciencia a fines económicos, aceptar innovaciones, perseguir los mejores materiales, consumir y tener hijos (en McClelland, 1968).

La necesidad de logro es uno de los factores psicológicos que mayor relación mantienen con el desarrollo económico. No obstante, para McClelland existen otros factores de naturaleza psicológica que también deben ser tenidos en cuenta. Este autor viene a decir que tras las situaciones y realidades de desarrollo socioeconómico de las diferentes regiones, sociedades y civilizaciones se encuentra un factor de enorme importancia, la necesidad de logro de sus integrantes. La necesidad de logro se traduce en la necesidad que tiene el individuo de superar estándares de excelencia con objeto de obtener satisfacción por sus consecuciones. De esta manera, un incremento de la necesidad de logro en una sociedad dada tendrá como resultado un mayor nivel de desarrollo económico y cultural.

El planteamiento metodológico original de McClelland consistía en establecer una relación empírica entre la necesidad de logro y el espíritu de empresa, ya que la creación de empresas es un mecanismo de indiscutible desarrollo para las regiones que las acogen. En otras palabras, se trataba de establecer una relación entre necesidad de logro y éxito en los negocios y aventuras empresariales al margen de los factores sociales, culturales e institucionales que presentaban los diferentes países con relación a su situación de desarrollo. Para ello se estudiaron más de setecientos empresarios pertenecientes a cuatro países -Estados Unidos, Turquía, Italia y Polonia-y se concluyó de manera empírica la hipótesis expuesta: "el móvil de logro constituye un importante factor que influye en el grado de desarrollo económico" (McClelland, 1968:45). Asimismo, el desarrollo cuantitativo de la investigación tuvo como resultado que las estimaciones realizadas sobre la necesidad de logro

\footnotetext{
${ }^{12}$ En el contexto de la gestión de Recursos Humanos, los aspectos "blandos" de la organización se refieren a los factores -competencias genéricas- sociales, culturales y psicológicos presentes en la organización. En otras palabras valores, creencias, actitudes, costumbres, motivaciones, expectativas, etc., constituyen el intangible de la empresa, pero, al mismo tiempo, son los factores más importantes y determinantes para su desarrollo y supervivencia.
} 
presentaran una correlación positiva con el desarrollo económico de esos países. En definitiva, quedó patente que la preocupación en torno a un estándar de excelencia, deseo expreso de tener éxito y creación de medios para conseguir metas ambiciosas eran variables que sin duda contribuían de manera representativa al desarrollo económico, pero que dicha relación -móvil de logro y logro económico-, a su vez, también depende de la estructura social y de las oportunidades que ésta permita (McClelland, 1968).

\section{LA INFLUENCIA DEL CAMBIO CULTURAL EN LOS PROCESOS DE DESARROLLO}

El fenómeno del cambio cultural está muy ligado al cambio social, político y económico. La cultura es un factor causal de enorme protagonismo en el actual entorno económico pero en la mayoría de los casos, como nos recuerda Inglehart, es menospreciado por la dificultad y complejidad en su medición, sobre todo a nivel cuantitativo. En palabras de este autor, "los factores culturales raramente se han medido cuantitativamente (...), hasta que no los cuantifiquemos y comprendamos, seguiremos en la oscuridad en lo que respecta a elementos cruciales del cambio social, político y económico" (Inglehart, 1991:51).

Para Inglehart, la relación entre cambio económico y cultural presenta una compleja causalidad recíproca, en la que los factores culturales no adoptan un papel pasivo que reciben los estímulos de los factores económicos, sino que también influyen en su naturaleza y desarrollo. Si bien son muchos los teóricos que defienden la vinculación existente entre cambios tecnológicos y económicos y cambios culturales y políticos, las discrepancias y debates comienzan cuando se plantea la direccionalidad de dicha interacción (Inglehart, en Díez Nicolás, 1994).

Partiendo de los datos de la Encuesta Mundial de Valores de 1990-91 (Díez Nicolás, 1994), se pueden establecer una serie de conclusiones en las que la relación existente entre cultura y desarrollo socioeconómico queda bastante patente. Las estructuras culturales coherentes ${ }^{13}$ existen y están vinculadas al desarrollo económico

\footnotetext{
${ }^{13}$ El término "estructuras culturales coherentes" hace referencia a la complementación de varios elementos culturales en un contexto de coherencia social. Ej. Gran importancia de la religión y existencia de familias numerosas.
} 
y tecnológico. Los resultados de la Encuesta Mundial de Valores se inclinan por la presencia de sistemas culturales condicionantes en sociedades en las que la coacción sociocultural supera al azar en cuanto a desarrollo socioeconómico se refiere. A su vez, el desarrollo económico conlleva transformaciones en el sistema de valores y creencias de la sociedad. Aun existiendo sociedades en las que las estructuras culturales son coherentes y su relación con el cambio tecnológico y económico es casi nula, la teoría de la modernización implica que el desarrollo económico está muy influenciado por determinadas estructuras culturales y que, a su vez, el factor cultural se ve afectado por el desarrollo socioeconómico. En otras palabras, los valores postmodernos están muy vinculados al desarrollo económico.

Bajo estos dos planteamientos se defiende la tesis de que la vivencia en un ambiente de prosperidad sin precedentes históricos y en ausencia de guerras, como ha sido el acontecido en los países occidentales desde 1945, ha hecho los más jóvenes valoren en menor proporción la seguridad física y económica, otorgen un menor valor a los aspectos materiales y se sientan más identificados con el sentido de comunidad y la calidad de vida (Inglehart, 1991). Siguiendo con estas tesis, la menor orientación materialista de esa cohorte de jóvenes se ha traducido en una reducción de la orientación al desarrollo socioeconómico y una mayor valoración de aspectos como la preservación del medio ambiente o la calidad de vida. Como se observa, los procesos de socialización iniciales en los que se ha visto inmerso el individuo, sumado a las vivencias y experiencias ajenas a la necesidad económica de primer orden, han dado lugar, junto con otras variables, a un menor desarrollo socioeconómico de la comarca, región y claro está, de las empresas y organizaciones que la integran.

\section{EL CASO ANDALUZ}

En el caso de Andalucía, a nuestro juicio, queda aún mucho por hacer a la hora de diseñar programas de intervención y desarrollo en el seno de una región y/o comunidad, ya que, tal y como se ha avanzando con anterioridad, se sigue invirtiendo poco en "cultura".

En el caso de Andalucía, los programas de desarrollo socioeconómico en general y de creación de empresas en particular, deben tener en cuenta estos factores a la hora de diseñar e implementar sus programas de intervención. Así, por ejemplo, 
los programas para el fomento del autoempleo, es decir, los destinados a incentivar y potenciar la creación de empresas, se siguen limitando a una serie de medidas de índole política, normativa y económica que no responden en absoluto al déficit emprendedor imperante en Andalucía, sujeto en la mayoría de los casos a la ausencia de una cultura emprendedora. En otras palabras se siguen limitando a la presencia de unos valores laborales que priorizan la seguridad, la estabilidad y la pasividad por encima de la iniciativa, la perseverancia, el riesgo o la proactividad, así como a la poca motivación por el logro existente entre la población activa. Nuestro planteamiento en este trabajo viene a manifestar que existe una importante relación entre cultura y desarrollo socioeconómico. Es decir, las diferentes formas y componentes culturales que conforman la sociedad andaluza ejercen una notable influencia en el desarrollo y evolución de sus estructuras socioeconómicas ${ }^{14}$.

Andalucía ha estado viviendo hasta hace muy poco un fuerte proceso de desvinculación productiva agraria, cuyas consecuencias culturales y sociales más importantes han determinado el cambio de identidad social y laboral de la región. Este tipo de desarrollo productivo ha determinado su modo de afrontar la vida y la existencia y, al mismo tiempo, ha dotado a la región de un peculiar universo simbólico que mantiene algunos de los valores de desarrollo propios del pasado ${ }^{15}$. En otras palabras, el enfrentamiento $o$ el conflicto de valores que encontramos constituye una principales causas que explican la situación socioeconómica actual de Andalucía ${ }^{16}$.

Es precisamente este componente antropológico, el simbolismo de las relaciones productivas, uno de los elementos más importantes que justifican el retraso socioeconómico que encontramos en Andalucía (Bericat Alastuey, 1989). En otras palabras, la propia imagen que de sí mismos han tenido los andaluces, más 0 menos real pero asimilada y autoimpuesta como real, ha sido uno de los principales obstáculos al desarrollo de la región. Así, la creencia asimilada del poco espíritu

${ }^{14}$ El desarrollo de las empresas, formadas por individuos y grupos, también está muy influenciado por los valores, creencias, normas y símbolos imperantes en su entorno.

${ }^{15}$ Ya en la década de los 80 , se pone de manifiesto la fuerte presencia de una motivación por el ahorro que no viene sino a corroborar el papel fundamental que ha jugado la seguridad en el sistema de valores de los andaluces, y que, por tanto, se ha traducido en un freno al desarrollo de la región (ESECA, 1987).

${ }^{16}$ Baste aquí recordar que una de las claves que relacionan la ética protestante con el surgimiento del espíritu capitalista se apoya en la caída de un sistema de valores tradicional y, en principio, poco favorecedor del desarrollo socioeconómico, y la aparición de otro sistema de valores más apropiado para hacer frente al cambio cultural existente. 
emprendedor, la escasa capacidad gerencial, la poca organización o la aversión al riesgo y a la innovación, se han convertido en etiquetas que han tenido mayor influencia que cualquier tipo de marginación financiera o comportamiento económico excluyente (Díez de Castro, 1995:23). Como nos apunta Díez de Castro, la situación socioeconómica de Andalucía se explica por "la existencia de pocas personas que presenten las cualidades indispensables para dar vida a una empresa” (1995:24).

Asimismo, tampoco debemos olvidar otro tipo de factores que, desde un punto de vista histórico, nos muestran un poco de luz a la hora de hablar de subdesarrollo en Andalucía. Aspectos como la carencia de una burguesía moderna, la sumisión de una clase media al servicio de una clase conservadora de origen terrateniente (Velarde, 1982), la suplantación del capital local por capital foráneo (Payno, 1983), la tradicional transferencia de ayudas que dio lugar a la ya conocida actitud pasiva, inmovilista y subsidiaria atribuida a la población andaluza, explican también gran parte de la situación de retraso económico de Andalucía.

\subsection{La Cultura Emprendedora en Andalucía}

El nacimiento y desarrollo de aventuras empresariales diseñadas con perspectiva de futuro y afincadas en un ecosistema empresarial imbricado en la cultura de la región generan sin lugar a dudas un alto nivel de desarrollo socioeconómico. Es por todos sabido que la iniciativa privada es una de las fuentes de desarrollo por excelencia (Guzmán Cuevas, 1987). Uno de los grandes hándicaps que encontramos en Andalucía es la ausencia de espíritu empresarial, más correctamente denominado como espíritu emprendedor o cultura emprendedora. Tradicionalmente, las comparaciones que se han realizado con otras comunidades autónomas como Cataluña o el País Vasco han arrojado datos muy importantes y significativos: desde un prisma cuantitativo el número de empresarios es drásticamente inferior en Andalucía. Una de las razones que se han utilizado para justificar esta falta de iniciativa empresarial y, ante todo, el "gap" existente entre la deseabilidad de crear una empresa y la realidad de hacerlo, radica en la falta de voluntad y mentalidad de logro en Andalucía (Bericat Alastuey, 1989). Tal y como nos recuerdan Vázquez y Sánchez (2000) en su libro Los Universitarios ante el trabajo, nos encontramos con que tan solo el $18 \%$ de los encuestados considera el autoempleo como una opción profesional, mientras que casi el $40 \%$ aspira a trabajar para el Estado. Ello no viene sino a consolidar la enorme presencia de una serie de aspectos culturales subyacentes que limitan el desarrollo de la iniciativa empresarial en nuestra comunidad. 
Al respecto, queremos dejar claro que estamos en total desacuerdo con aquellos que asemejan autoempleo y espíritu emprendedor ya que entendemos que para montar una empresa no hace falta ser emprendedor, y viceversa, se puede ser emprendedor sin necesidad de crear una empresa. Es más, estamos asistiendo a una enorme tasa de mortalidad empresarial ${ }^{17}$ en Andalucía, es decir, empresas que se ven obligadas a cerrar en sus primeros años, como consecuencia, entre otras, de la ausencia de una cultura emprendedora sólida y arraigada, así como de unos valores coherentes con la misma. La empresa andaluza presenta unos importantes déficits en cuanto a la presencia de una cultura emprendedora -dinamismo, riesgo, proactividad, planificación, iniciativa, logro, etc.,- y una orientación al logro. Reorientemos estas pautas culturales, psicosociales y motivacionales y mejoraremos la calidad y cantidad de nuestro tejido empresarial.

Como ya se ha visto en apartados anteriores, la relación existente entre móvil de logro y creación de empresas es muy alta. Es más, muchas de las medidas que desde diferentes organismos e instituciones se han desarrollado para fomentar la creación de empresas tienen entre sus principales receptores aquellas personas que crean una empresa como alternativa o soluciona su problema de desempleo, irregularidad o precariedad laboral olvidando que -en palabras de Bericat Alastuey- podemos encontrarnos con la proliferación de empresas-chabolas, es decir, empresas en las que la falta del conocimiento de gestión empresarial y la falta de espíritu emprendedor es una constante en la corta vida de la empresa. En otras palabras, ni todos los empresarios son emprendedores ni todos los emprendedores son empresarios.

A este respecto, hacemos referencia a la Encuesta Mundial de Valores para el caso de Andalucía (Pino Artacho y Bericat Alastuey, 1998), en la que los datos ponen de manifiesto una mayor presencia de valores "postmaterialistas" entre la población andaluza ${ }^{18}$. Como consecuencia de la inseguridad e inestabilidad del sistema económico y laboral andaluz, se observa una mayor valoración de la seguridad en los niveles económico y social. Como se observa, los valores de la estabilidad

\footnotetext{
${ }^{17}$ Así, por ejemplo, en la provincia de Granada, desde 1997 se han constituido un total de 8.548 empresas, de las cuales, en el periodo que va de 1997 a marzo de 2002, 269 se han extinguido, 536 se han disuelto, 15 se han escindido, 16 se han fusionado, 4 han quebrado y 5 se han suspendido (Fuente: Axesor Grupo Infotel, 2002).

${ }^{18}$ Los resultados del estudio muestran que el $64,5 \%$ se inclina por los valores de la seguridad, el mantenimiento del orden y las metas económicas, mientras que el 33,1\% de la población encuestada valora en mayor medida la participación, la libertad de expresión o una sociedad más humana.
} 
y regularidad chocan radicalmente con el entorno económico, social y laboral, cambiante, flexible e impredecible en el que vivimos en la actualidad, y, sobre todo, con el desarrollo de la aventura empresarial, caracterizada por el riesgo, la flexibilidad, el cambio, el dinamismo y movimiento constantes. En definitiva, esta realidad cultural se traduce a su vez en un menor desarrollo de nuestras empresas, en una reducción de su eficiencia y competitividad, en un menor número de nuevas empresas, y, en definitiva, en un menor desarrollo socioeconómico de la región andaluza.

\section{CONCLUSIONES}

Crecimiento económico y empleo constituyen dos factores indisociables y de naturaleza sinérgica. Para incrementar la base productiva andaluza deben multiplicarse los estudios que presentan los nuevos yacimiento de empleo, ya que orientan hacia dónde deben dirigirse los procesos, programas e itinerarios formativos, pero al mismo tiempo deben modernizarse los referentes laborales de la población activa en el sentido de fomentar, sensibilizar y concienciar a este colectivo sobre las ventajas que presentan los patrones sociolaborales emprendedores y empresariales (Galindo, en Prior, Hita Alonso y otros, 2002).

La situación socioeconómica de Andalucía en el nuevo milenio pasa, entre otras, por la revalorización y potenciación de la iniciativa privada. La realidad empresarial se torna clave en el despegue del empleo en Andalucía y, por tanto, deben florecer alternativas de investigación-acción que fomenten el desarrollo de nuevas empresas y garanticen el desarrollo y la competitividad de las ya existentes. Asimismo, la región andaluza ofrece múltiples sub-realidades que no conviene olvidar, en concreto, en lo referente a la situación socioeconómica y laboral de Andalucía oriental y occidental, $y$, por tanto, hay que profundizar en cada una de ellas con objeto de trazar medidas correctoras adecuadas a la situación de cada provincia o sector regional.

A este respecto, y como ha se ha adelantado en anteriores epígrafes, una de las grandes problemáticas laborales de Andalucía radica en la cultura productiva imperante en la que han reinado una serie de valores sociales y laborales, en principio, contrarios al desarrollo de la aventura empresarial. La figura del emprendedor es de vital importancia para el desarrollo; es más, de él depende en gran medida el grado de bienestar económico y social de las regiones (Prat Ballester, 1986). Pero a nuestro juicio, emprendedor no es solo aquella persona que monta una empresa, sino también 
aquel trabajador por cuenta ajena que presenta valores de dinamismo, iniciativa, riesgo, creatividad o independencia. Uno de los grandes problemas de Andalucía radica no tanto en la ausencia de emprendedores -que los hay, en el sentido de montar una empresa-, sino la falta de una cultura emprendedora, que tiene como nefastas consecuencias, entre otras, las altas tasas de mortalidad empresarial (Díez de Castro, 1995). Como nos ha recordado hace poco el famoso "banquero de los pobres", Muhammad Yunus, el autoempleo es fundamental para el desarrollo de una región pero hay que tener mucho cuidado con las subvenciones o la ya denominada "cultura de la subvención", ya que éstas limitan en la gente la responsabilidad y la iniciativa, que es la parte más esencial del ser humano (Yunus, 2002:4). Para Yunus, las subvenciones solo tienen sentido en casos excepcionales y de forma temporal-un desastre, es decir, por causas externas e impredecibles-, es más, las subvenciones nunca deben ser permanentes para nadie porque, como se ha dicho, limitan la responsabilidad y la iniciativa del emprendedor.

Como se ve, son muchas las virtudes y potencialidades de la economía andaluza, pero, al mismo tiempo, están demasiado presentes una serie de factores que obstaculizan y coartan el desarrollo socioeconómico de la región. El factor más problemático es sin duda la falta de un empleo estable y duradero, pero, para su resolución, no basta con el diseño e implementación de medidas de índole política y/o económica, sino que se presenta como de vital importancia el cambio de los valores laborales, así como de la mentalidad y de los procesos de socialización laboral imperantes, para que en un futuro a medio plazo la asunción de riesgos empresariales y la iniciativa empresarial sean consideradas como prácticas y alternativas propias de nuestro entorno económico y sociolaboral.

Esperamos que el presente trabajo haya servido para concienciar al/la lector/a de la existencia de una realidad socioeconómica cada vez más compleja e "irracional" en la que los componentes soft de la vida social están cada vez más presentes. Así, invitamos a todos aquellos estudiosos e investigadores de la realidad económica y empresarial en Andalucía a que inicien nuevas vías de intervención en las que la variable cultural, junto con las de naturaleza económica y política, jueguen un papel clave en los diseños y reflexiones que sobre la misma se llevan a cabo. Solo de esta manera podremos mejorar los niveles de desarrollo económico, tan necesarios y claves en la existencia y crecimiento de muchos pueblos, comarcas y provincias de nuestra región. 


\section{BIBLIOGRAFÍA}

ADELMAN, I. (1974). Teorías del desarrollo económico. Fondo de cultura económica. México D.F.

ALMOND, G.A. Y VERBA, S. (1970). La cultura cívica. FOESSA. Madrid.

ARGANDOÑA, A., BUCHANAN J., STREISSLER, E. Y OTROS (1994). Economía y dinámica social. Reflexiones acerca de la acción humana ante un nuevo ciclo histórico. Universidad Pontificia Comillas. Unión Editorial. Madrid.

BUCHANAN, J.M. (1995). Etica y progreso económico. Colección de Estudios e Informes. Num.3. Servicio de Estudios. La Caixa. Madrid.

CAÑADILLA GÓMEZ, M. (2000). Manual-Guía para el fomento de la Cultura ÉticoEmprendedora (E2). Fondo Formación. Granada.

DANEKE, G. (1998). "Beyond Schumpeter: Nonlinear economics and the evolution of the U.S. innovation system", Journal of Socio-Economics, vol 27, $n^{0} 1$.

DAHRENDORF, R. (1983). Oportunidades vitales. Espasa-Calpe. Madrid.

DÍAZ, A. Y KAWAMURA, Y. (1994). La cultura empresarial japonesa en España. Madrid. Cívitas. Madrid.

DÍEZ DE CASTRO, E., GALÁN, J,L., Y LEAL, A. (1995). La empresa en Andalucía. Cívitas. Madrid.

DÍEZ NICOLÁS, J. E INGLEHART, R. (1994). Tendencias mundiales de cambio de valores sociales y políticos. Fundesco. Madrid.

ECHEVARRI SUVERBIOLA, A. (1988). Introducción a la cultura económica. Ciencias de la Dirección. Madrid.

FROMM, E. (1974). Sociopsicoanálisis del campesino mexicano. FCE. México.

GALINDO CALVO, P. (2003). La Cultura Empresarial en Andalucía. Estudio sociológico de la pequeña empresa en Granada capital. Tesis Doctoral. Universidad de Granada (Pendiente Publicación) 
GUZMAN CUEVAS, J. (1987). "El desarrollo económico-social de Andalucía". Revista de Estudios Andaluces. № 18.

HARRISON, L. ( 1987). El subdesarrollo está en la mente. Playor. Madrid.

INGLEHART, R. (1991). El cambio cultural en las sociedades industriales avanzadas. CIS. Siglo XXI. Madrid.

INGLEHART, R. (1997). Modernization and Posmodernization. Cultural Economic and Political Change in 43 Societies. Princeton, Princenton University Press.

MCCLELLAND, D.C. (1961). La sociedad ambiciosa. Guadarrama. Madrid.

MCCLELLAND, D.C. (1973). "Testing for competence rather than for intelligence". American Psychologist, 28, 1-14.

PAYNO, J.A. (1983). Claves para el desarrollo económico de Andalucía: Informe de la Junta de Andalucía sobre las potencialidades económicas de Andalucía en la perspectiva de la entrada en la CEE. Junta de Andalucía. Sevilla.

PARSONS, T. (1982). El sistema social. Occidente. Madrid.

PICÓ, J. (2000). Cultura y modernidad. Seducciones y desengaños de la cultura moderna. Alianza Editorial. Madrid.

PINO ARTACHO, J.del y BERICAT ALASTUEY, E. (1998). Valores sociales en la cultura andaluza: encuesta mundial de valores: Andalucía 1996. Centro de Investigaciones Sociológicas. Siglo XXI de España. Madrid.

PRAT BALLESTER, J. (1986). El emprendedor hoy. Hispano-Europea. Barcelona.

REQUENA SANTOS, F. (1999). Sociedad, cultura y desarrollo. Universidades de Málaga y Almería.

RITZER, G. (1993). Teoría Sociológica Contemporánea. McGraw-Hill. Madrid.

VALLÉS FERRER, J. (1997). Economía andaluza. Algaida. Sevilla. 
VELARDE, J. (1982). Decadencia y crisis en Andalucía. Instituto de Desarrollo Regional. Sevilla.

VERNON, P.E. y FORTEZA MÉNDEZ, J.A. (1980). Inteligencia y entorno cultural. Marova. Madrid.

WEBER, M. (1997). La ética protestante y el espíritu del capitalismo. Península. Barcelona.

YUNUS, M. (2002). "El banquero de los pobres". Diario IDEAL, 7 de febrero de 2.002. Granada. 